\title{
The Research of Key Technologies of Streaming Media Digital Resources Transmission Based on CDN and P2P
}

\author{
Jichun Zhao ${ }^{1, *}$, Feng Yu ${ }^{1}$, Junfeng Zhang ${ }^{1}$, Sufen Sun ${ }^{1}$, \\ Jianxin Guo ${ }^{1}$, Jing Gong ${ }^{1}$, and Yousen $\mathrm{Zhao}^{2}$ \\ ${ }^{1}$ Institute of Information on Science and Technology of Agriculture, \\ Beijing Academy of Agriculture and Forestry Sciences, Beijing, China, 100097, \\ Tel.: 86-010-51503172 \\ zhaojichun_0@163.com \\ ${ }^{2}$ The Information Center of Beijing Municipal Bureau of Agriculture, Beijing, China
}

\begin{abstract}
Traditional system based on the current form of distance education network technology does not support the existence of dynamic multimedia data and real-time streaming data, as well as the existence of streaming media technology in the server bottleneck problem. The key technologies of streaming media digital resources transmission based on CDN (Content Delivery Network) and P2P (peer-to-peer) is discussed in the paper, and the P2P core algorithm is optimized, the CDN network deployment of the program and the edge server deployment strategies are discussed, which can address the situation of network congestion and improve user access to video and web site response time.
\end{abstract}

Keywords: P2P, CDN, Steaming media.

\section{Introduction}

Multimedia Network teaching now begins becoming a most important teaching way besides the traditional teaching way. This new teaching way aims at that anyone can learn anything at any time and from anywhere. Facing with the very large amount of multimedia data, many technologies should be involved in and variety of computer resources should be utilized if you want to transmit multimedia data efficiently on the internet.

Peer-to-peer is a communications model in which each party has the same capabilities and either party can initiate a communication session. Other models with which it might be contrasted include the client/server model and the master/slave model. In some cases, peer-to-peer communications is implemented by giving each communication node both server and client capabilities. In recent usage, peer-to-peer has come to describe applications in which users can use the Internet to exchange files with each other directly or through a mediating server.

On the Internet, peer-to-peer (referred to as P2P) is a type of transient Internet network that allows a group of computer users with the same networking program to

\footnotetext{
Corresponding author.
} 
connect with each other and directly access files from one another's hard drives. Major producers of content, including record companies, have shown their concern about what they consider illegal sharing of copyrighted content by suing some P2P users.

Meanwhile, corporations are looking at the advantages of using P2P as a way for employees to share files without the expense involved in maintaining a centralized server and as a way for businesses to exchange information with each other directly.

Short for content delivery network, a network of servers that delivers a Web page to a user based on the geographic locations of the user, the origin of the Web page and a content delivery server. A CDN copies the pages of a Web site to a network of servers that is dispersed at geographically different locations, caching the contents of the page. When a user requests a Web page that is part of a CDN, the CDN will redirect the request from the originating site's server to a server in the CDN that is closest to the user and deliver the cached content. The CDN will also communicate with the originating server to deliver any content that has not been previously cached.

This service is effective in speeding the delivery of content of Web sites with high traffic and Web sites that have global reach. The closer the CDN server is to the user geographically, the faster the content will be delivered to the user. CDN also provide protection from large surges in traffic. The process of bouncing through a CDN is nearly transparent to the user. The only way a user would know if a CDN has been accessed is if the delivered URL is different than the URL that has been requested.

A peer-to-peer (P2P) Contents Delivery Network $(\mathrm{CDN})$ is a system in which the users get together to forward contents so that the load at a server is reduced. Lately, we have high-speed services for an access to the Internet such as the Asymmetric Digital Subscriber Line (ADSL). Some broadcasters may not have such services because they have only dial-up services and wireless services as PHS and a mobile phone to broadcast live. A problem with P2P CDN is its overhead to construct a distribution tree. It becomes a crucial problem when a broadcaster has only a low-speed access to the Internet, and we propose a P2P CDN system which reduces such an overhead. The key technologies of streaming media digital resources transmission based on CDN and P2P is discussed in the paper, and the P2P core algorithm is optimized, the CDN network deployment of the program and the edge server deployment strategies are discussed, which can address the situation of network congestion and improve user access to video and web site response time.

\section{The Research Content}

Web server based on the current form of distance education network technology does not support the existence of dynamic multimedia data and real-time streaming data, as well as the existence of streaming media technology in the server bottleneck problem. The key technologies of streaming media digital resources transmission based on $\mathrm{CDN}$ and $\mathrm{P} 2 \mathrm{P}$ is discussed in the paper, and the $\mathrm{P} 2 \mathrm{P}$ core algorithm is optimized, the $\mathrm{CDN}$ network deployment of the program and the edge server deployment strategies are discussed, which can address the situation of network congestion and improve user access to video and web site response time.

The key technology of P2P transfer research is that the core algorithm of $\mathrm{P} 2 \mathrm{P}$ optimization to study the domain to support the autonomy of the P2P discovery and 
optimization of management agreements, so that the impact of interactive $\mathrm{P} 2 \mathrm{P}$ networks to the whole net can be decrease minimum. P2P streaming media transmission node in the core agreement in accordance with $\mathrm{P} 2 \mathrm{P}$ content (including files and streams) to deal with so slices, $\mathrm{P} 2 \mathrm{P}$ users will be in accordance with these rules to complete the $\mathrm{P} 2 \mathrm{P}$ sharing. The edge server pressure can be reduced greatly, and the file transfers and streaming media transmission can be improved efficiency.

CDN network deployment optimization program is to study the edge server deployment strategies, in order to solve the situation of network congestion problem and improve respond speed of user access to the site. The main technologies include distributed storage, load balancing, request redirection network and content management such as the core technology, and content management and global management of network traffic (Traffic Management) is the core of CDN. Through the user and the server load the nearest judge, CDN to ensure that the contents in a very efficient way for users to request services, which can be achieve fast and redundant for the purpose of accelerating the multiple sites.

\section{The Research Objective}

The text is research on $\mathrm{P} 2 \mathrm{P}$ streaming media distribution system architecture, and establishment of P2P streaming media distribution model, in order to address the limited bandwidth which can not meet the increasing number of customer service demand. The key technology of P2P transfer research is that the core algorithm of P2P optimization to study the domain to support the autonomy of the P2P discovery and optimization of management agreements. CDN network deployment optimization program is to study the edge server deployment strategies, in order to solve the situation of network congestion problem and improve respond speed of user access to the site.

Research on key technologies of the P2P streaming media live, and the test system of $\mathrm{CDN}+\mathrm{P} 2 \mathrm{P}$ based on streaming media is set up, analysis and test the deployment of $\mathrm{P} 2 \mathrm{P}$ algorithms and programs $\mathrm{CDN}$.

\section{The Key Technology}

\subsection{Research on P2P Streaming Media Distribution System Architecture}

Research on P2P streaming media distribution system architecture provides a solution to the current distribution of streaming media in a limited bandwidth.

The paper is research on $\mathrm{P} 2 \mathrm{P}$ streaming media distribution system architecture which uses the existing CDN network. The user side of the edge server is as a superseed provider, and at the same time user terminals provide the media distribution, content storage and effective management of resources, which can improve Unicast Quality of Service. P2P traffic will be severely restricted in the same region of the edge node through the distribution hybrid structure in order to avoid the flow hedge in the backbone of the Internet, and which increased manageability and high reliability of service. 


\subsection{P2P Live Streaming Media Key Technologies}

Using member services and certificate realize building peer group and joining the inspection. Streaming media will not be affected and increased the capacity of the system through multi-source transmission and node monitoring service quality in the P2P network nodes dynamically changing environment. The P2P live streaming key is building a $\mathrm{P} 2 \mathrm{P}$ network, data transmission, security and issues such as QoS guarantee.

\subsection{P2P Cache Strategy and Replacement Algorithm}

The technology advance in network has accelerated the development of multimedia applications over the wired and wireless communication. To alleviate network congestion and to reduce latency and workload on multimedia servers, the concept of multimedia proxy has been proposed to cache popular contents. Caching the data objects can relieve the bandwidth demand on the external network, and reduce the average time to load a remote data object to local side. Since the effectiveness of a proxy server depends largely on cache replacement policy, various approaches are proposed in recent years.

A proxy capable of transcoding is called transcoding proxy. Since the transcoding proxy lies between the server and the clients, this architecture can coordinate the mismatch between what the server provides and what the client prefers. The proxybased approach has several advantages. First, an intermediate proxy has larger bandwidth to download the web objects and higher computing power to perform encoding/decoding, which enables proxy-based transcoding to effectively reduce the

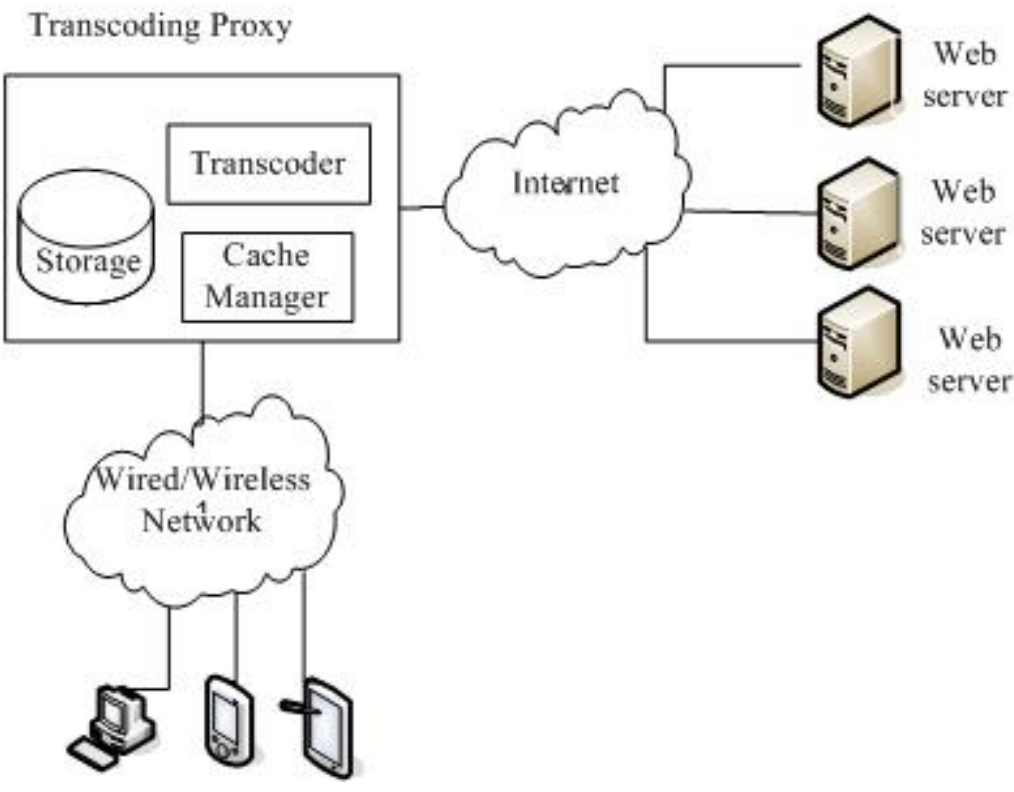

Fig. 1. The transcoding proxy structure 
waiting time for clients to access the required version. Moreover, the proxy-based technologies can perform on-the-fly transcoding for dynamic content adaptation. Therefore, the proxy-based architecture combines the advantageous features of the client-based and server-based technologies. Figure.1 shows the architecture of the transcoding proxy. There are two primary components: transcoder and cache manager. After downloading the web objects from the remote servers, the transcoder should perform transcoding according to the clients' requirements. The transcoding results will be kept in the local storage (referred to as cache) of the proxy. Since the cache has limited capacity, the cache manager will perform cache replacement to evict the less valuable objects. Research topics in proxy-based transcoding include cache replacement schemes, system architectures, and proxy collaboration.

\section{The Research Program -P2P + CDN Streaming Media Broadcast Model}

\subsection{System Function}

Living channel list can be generated in the web page, a user clicks on different channels, the can watch different living channel programs.

According to the specific needs, the user can freely set the living parameters. The parameters are the size of the video window, video stream, and video encoding format. Real-time video surveillance can monitor the living content.

It can be set in the process of living access policy control, when a user exceeds the maximum on-line number of simultaneous users, other user access to be controlled.

\subsection{The Flow of Living}

The living flow: real-time satellite video programming or other video source in real time by the encoder to code for high-definition H.264 format video streams sent to the programs origins of media servers, media server shows the distribution of the origins of the program will be broadcast live to push down broadcast server, as well as programs can be sent to the program library for storage, the server may be broadcast live programming direct broadcast simultaneously live broadcasts can also be distributed CDN servers, CDN servers will be live broadcasts push terminals;

Living initiative launched by the terminal, terminal-demand programming flow: End users first need to log in and teaching web portal management system authentication, identity verification through after clicking on the appropriate program to receive the video on demand, terminal intelligent in choosing the best CDN server (recently and optimal server) to receive data streams.

End-user log in at teaching management system and click on programs will be teaching management at the server log information to do records, Report information to generate statistics in order to study the process of timely feedback. The structure of living is shown as Fig.2. 


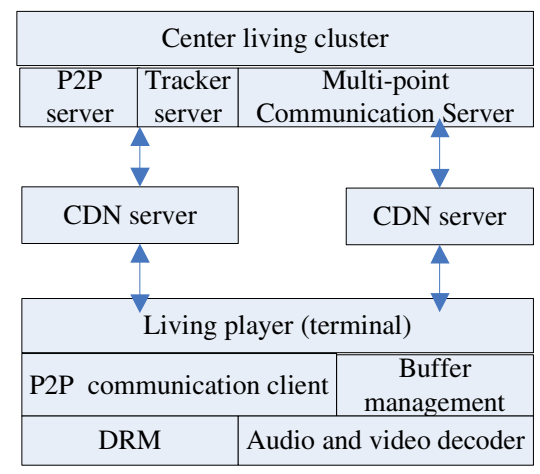

Fig. 2. The structure of living

\section{The Test Effect}

The video plays clearly, through its strong enabling technology to provide users with a more perfect enjoyment of the audio-visual situation and there is no buffer.

The drag response speed is fast, to enable users to select video was the moment, the system immediately begin to watch the speed of the user at least 3 times the speed of download videos and simultaneously broadcast buffer situation does not arise.

Living response is fast, the user at the request of streaming media services and switch channels hardly feel the delay, but sound and video always maintain smooth continuous, not a reproduction pause, such as jitter and discontinuous phenomenon.

Facilitate management: Modular design and fully technology application development, can achieve more than the business aspect as well as support and content providers (ICP) into programs such as outsourcing, co-operation of encryption programs, video streaming, data flow, cost flow interaction, the real implementation seamlessly between the various systems used in combination.

\section{The Direction of Future Research}

First of all, the need for further research question is how to generate effective coverage of P2P application-layer topology. Systems not only need to consider scalability and fault-tolerant, and also be taken into account the dynamic nature of nodes and heterogeneity, and in accordance with the status of physical network, and dynamically adjusted to optimize the topology and error handling. At the same time, minimize system maintenance costs in order to improve network utilization.

Security issues of P2P streaming media technology, one of research directions. How to node authentication, digital signature for the content providers and, in conjunction with digital rights management DRM technology to ensure the legitimacy of media content, whether for the protection of user privacy from being violated, or for P2P Streaming Media Technology Application and Promotion, has importance.

Combine Network coding P2P technology and streaming media technology to increase the calculation of the volume of network nodes for improving the network 
throughput and enhancing system robustness and stability has high research value and use value.

\section{References}

Hung, H.-P., Chen, M.-S.: Maximizing the profit for cache replacement in a transcoding proxy. In: Proceedings of IEEE ICME (2005)

Castro, M., Druschel, P., Kermarrec, A.: Metal. SCRIBE: A large-scale and decentralized application-level multicast infrastructure. IEEE JSAC 20(8) (October 2002)

Castro, M., Druschel, P., Kermarrec, A.: Metal. SplitStream: High Bandwidth content distribution in cooperative environments. In: IPTPS'0, Berkeley, CA (February 2003)

Tran, D., Hua, K., Do, T.: Zigzag: An efficient peer-to-peer scheme for media sreaming. In: Proc.of the IEEE IN FOCOM 2003, pp. 1283-1293. IEEE Computer and Communications Societies, NewYork (2003)

Hefeeda, M., Habib, A., Botev, B., Xu, D., Bhargava, D.B.: PROMISE:A peer-to-peer media streaming using collect cast. In: Proc.of the ACM Multimedia 2003, pp. 45-54. ACM Press, New York (2003)

Ford, B., Srisuresh, P.: Peer-to-Peer communication across iddleboxes, http: //midcom-p2p. sourceforge.net/

draft-ford-midcom-p2p-Ol.txt

Syverson, P., Reed, M., Goldschlag, D.: Onion Routing Access Configurations. In: Proceedings of DARPA Information Survivability Conference and Exposition (DI: SCEX 2000), pp. 34-40 (2000)

Abrams, M., Standridge, C.R., Abdulla, G., Williams, S., Fox, E.A.: Caching proxies: limitations and potentials. In: Fourth International World-Wide-Web Conference (1995)

Han, R., Bhagwat, P., LaMaire, R., Mummert, V.P.T., Rubas, J.: Dynamic adaptation in an image transcoding proxy for mobile www browsing. IEEE Personal Communication (December 1998)

Padmanabhan, V., Wang, H., Chou, P.: Distilbuting Streaming Media Content using Cooperative Networking. In: Proc. of the imitational workshop on network and operation system support for digital audio and video, Miami, Florida (2002) 